\title{
La puella venenata entre littérature et anthropologie
}

\section{Carlo Donà}

Traducteur : Silvia Disegni

\section{OpenEdition \\ Journals}

Édition électronique

URL : http://journals.openedition.org/recherchestravaux/590

DOI : 10.4000/recherchestravaux.590

ISSN : 1969-6434

\section{Éditeur}

UGA Éditions/Université Grenoble Alpes

\section{Édition imprimée}

Date de publication : 15 mai 2013

Pagination : 141-158

ISBN : 978-2-84310-248-6

ISSN : 0151-1874

\section{Référence électronique}

Carlo Donà, «La puella venenata entre littérature et anthropologie », Recherches \& Travaux [En ligne], 82 | 2013, mis en ligne le 15 novembre 2014, consulté le 08 septembre 2020. URL : http:// journals.openedition.org/recherchestravaux/590 ; DOI : https://doi.org/10.4000/recherchestravaux. 590

(c) Recherches \& Travaux 
Carlo DonÀ

Université de Messine, Italie

\title{
La puella venenata entre littérature et anthropologie
}

\author{
(Traduit de l'italien par Silvia Disegni)
}

I. Les pages qui suivent traitent la question de la puella venenata, que nous pourrions résumer par cette formule : jusqu'à quel point les femmes peuvent-elles être vraiment venimeuses? Il s'agit d'un thème vaste, ancien et fatalement un tantinet épineux, peu connu et peu étudié aujourd'hui, mais toujours vivant dans la culture populaire. Ceux qui se rappellent le personnage fascinant et mortifere de Poison Ivy dans Batman and Robin de Joel Schumacher (1997), interprété par une remarquable Uma Thurman, savent déjà l'essentiel. En synthèse, la puella venenata est une femme, en général très belle, qui devient mortifere, par nature ou à la suite d'un accident, et qui unit donc en soi Éros et Thanatos en un développement aussi irrésistible que fatal, car elle inflige généralement la mort à travers un acte amoureux : comme Poison Ivy, elle tue en embrassant quelqu'un ou en faisant l'amour avec lui.

Ce thème plutôt négligé par les études a été magistralement mis en lumière voilà bien longtemps - en 1893 - dans un article fondamental de Wilhelm Hertz, véritable chef-d'œuvre de la grande philologie allemande de la seconde moitié du XIX ${ }^{e}$ siècle, qui réunissait tout le dossier sur la puella venenata, en associant avec un naturel parfait les perspectives philologique et anthropologique ${ }^{\mathrm{r}}$. Ces deux orientations se sont ensuite scindées, si bien que

I. W. Hertz, "Die Sage vom Giftmädchen", Abhandlungen der bayerischen Akademie der Wissenschaften, I893, p. 89-166, puis dans Gesammelte Abhandlungen von Wilhelm Hertz, éd. Fr. von der Leyen, I905, p. I56-277. Ce thème a été repris ensuite par Penzer dans sa grande édition du Kathāsaritsägara: The Ocean of Story, being C. H. Tawney's Translation of Somadeva's Kathā sarit sägara (or Ocean of Stream of Story), nouv. éd. N. M. Penzer, vol. II, Londres, Chas. J. Sawyer, I924, appendix III, Poison-Damsels, p. 275-313 (réimprimé ensuite 
dans les dernières études consacrées à ce sujet - respectivement, par un philologue, Claude Thomasset, et par une anthropologue, Gillian Bennett ${ }^{2}-$, elles paraissent désormais si éloignées l'une de l'autre qu'elles sont devenues pratiquement opposées' C'est bien dommage : car c'est seulement à partir d'une perspective stéréométrique, à la fois littéraire - c'est-à-dire historique et philologique - et anthropologique, que l'on pourra expliciter correctement les dynamiques culturelles qui sous-tendent et qui génèrent notre thème.

2. J'ai dit, en mentionnant Poison Ivy, que le thème de la puella venenata est bien vivant dans la culture actuelle. J'aurais pu mentionner bien d'autres figures, comme le band anarchopunk des Poison Girls; ou plusieurs des personnages des mangas japonais, telle Kagero de Ninja Scroll; ou encore Rogue, dans $X-M e n$. Pour des palais plus raffinés, je pourrais citer la Beatrice Rappaccini de la nouvelle de Hawthorne (I844), The Poison Maid de Richard Garnett (I888), éventuellement dans la version opéra de Vaughan Williams (The Poisoned Kiss, représenté pour la première fois en 1936), ou même le grand livre qu'est The Real Life of Sebastian Knight de Vladimir Nabokov (194I), qui est certainement redevable à notre histoire.

L'archétype de cette constellation culturelle composite est très ancien : géographiquement, il voit le jour dans le Nord de l'Inde et, chronologiquement, il est bien antérieur à l'An Iooo, et peut-être même à l'époque vulgaire. Une série de textes indiens dont les intrigues se déroulent à l'époque de la formation du premier empire Maurya, nous présentent en effet la višakanyā ("Giftmädchen») o višängunā, ("Giftweib»)", qui est une sorte d'aimable killer, pour le dire en peu de mots, car il s'agit d'une fille, très belle, élevée

dans N. M. Penzer, Poison-Damsels and Other Essays in Folklore \& Anthropology, Chas. J. Sawyer, Londres 1952, rpt. New York, Arno Press, 1980, p. 3-73). Signalons aussi les études remarquables, mais désormais oubliées, de J. J. Modi, «The Vish-Kanya or Poison - Damsels of Ancient India, Illustrated by the Story of Susan Ramashgar in the Persian Burzo-Nameh ", Folklore, $\mathrm{n}^{\circ} 4$ (déc. 3I, 1927), vol. 38, p. 324-337; du même, "The story of Alexander the Great and the Poison-Damsel of India", Journal of the Royal Asiatic Society - Bombay Branch, I928, vol. III, p. 212-230.

2. Cl. Thomasset, Une vision du monde à la fin du XIII siècle, commentaire du dialogue de Placides et Timéo, Genève, Droz, 1982, p. 69-108; G. Bennet, Bodies. Sex Violence, Disease and Death in Contemporary Legend, Jackson, University of Mississippi Press, 2005.

3. L'étude de Thomasset est vaste, bien documentée et rigoureuse, mais entièrement limitée au passé et sans la moindre ouverture sur la modernité; celle de Gillian Bennet a le mérite d'identifier nombre de connexions intéressantes, mais elle a tous les défauts - pour moi extrêmement irritants - de l'anthropologie anglo-américaine : elle manque absolument de perspective historique, elle fait systématiquement des clins d'œil au marché et, surtout, elle ignore complètement tout ce qui n'est ni moderne ni écrit en anglais, et, en particulier, le texte de Hertz.

4. W. Hertz, ouvr. cité, I893, p. 236. 
pour tuer, comme le raconte Zakariyyāi al-Qazwīnī (I2O2-I283) dans son 'Ajäib al-makhlūqat wa-gharäib al-mawjüdāt ("Merveilles des Créatures et des choses étranges qui existent»).

Parmi les merveilles de l'Inde, on peut mentionner la plante el-bîś, qui se trouve seulement en Inde et qui est un poison mortel. On dit que lorsque les rois indiens veulent conquérir un souverain ennemi, ils prennent une petite fille à peine née et mettent d'abord la plante quelque temps sous son berceau, puis sous son matelas et enfin sous ses vêtements. Enfin, ils lui donnent à boire du lait dans lequel la plante a été mise à macérer, jusqu'à ce que l'enfant puisse la manger sans danger. Puis, cette fille, ils l'envoient en cadeau au roi qu'ils veulent détruire et quand celui-ci s'unit à elle, il meurts .

Le nom de la plante el-biś provient du sanskrit viša, qui désigne surtout l'Aconitum napellus, une plante particulièrement belle mais extraordinairement vénéneuse, au point qu'il peut même être dangereux d'en tenir une branche dans sa main : ce terme a donné par dérivation višakanyā et višāngunā (dans lequel nous reconnaissons le grec gyné).

Al-Qazwīnī se réfère dans ce passage à une coutume qui nous est connue grâce à beaucoup d'autres sources indiennes, surtout littéraires, comme le Mudrā-Rākshasa di Visakhadatta, une œuvre théâtrale dont la datation est très incertaine (entre le $\mathrm{IV}^{\mathrm{e}}$ et le $\mathrm{VII}^{\mathrm{e}}$ siècle) et narre la montée au trône du grand Chandragupta (mort en 298 avant J.-C.), le Sandrokottos ou Sandracotto des sources occidentales. Nanda, roi de Magadha, et son premier ministre Rakshasha, tentent à plusieurs reprises d'éliminer Chandragupta, qui est systématiquement sauvé par l'astuce de son conseiller Chanakya. En particulier, Rakshasha envoie à Chandragupta une višakanyā, qui est toutefois reconnue comme telle et donc envoyée en cadeau à un allié de Chandragupta devenu encombrant, le roi Parvataka, qui tombe aussitôt victime du charme toxique de la jeune fille. On trouve d'autres versions de cette histoire dans le Parisistaparvan de Hemacandra (I089-II72), dans le Kathāsaritsāgara de Somadeva et dans d'autres textes qui n'ont pas nécessairement un caractère littéraire : ainsi, cette histoire a également laissé des traces dans la tradition astrologique, laquelle spécifie dans quelles configurations astrales doivent naître les petites filles destinées à jouer ce rôle néfaste.

Il vaut la peine d'ajouter quelques gloses en marge de ce premier groupe de récits sur notre thème, en soi plutôt intéressants.

I) Pour ce que nous comprenons des textes, toujours curieusement réticents, la jeune fille est empoisonnée progressivement avec une substance

5. En l'absence d'une meilleure édition, je tire le texte de Z. i. M. Al-Qazwinī, Kosmographie, dans S. de Sacy, Chrestomathie arabe [I806], Paris, Imprimerie impériale, vol. III, p. 398 (extrait traduit en français par l'auteur de l'ouvrage). 
végétale, généralement identifiable comme étant l'aconit. Elle empoisonne par contact - comme c'est précisément le cas aussi avec l'aconit -, mais pas exclusivement à travers un contact sexuel (le Parisistaparvan, par exemple, précise que Parvata est intoxiqué quand il la tient par la main), et, semble$\mathrm{t}$-il, elle ne fonctionne qu'une seule fois.

2) Les mentions de la višakanyā sont rares et sommaires; on a toutefois l'impression que la pratique en soi était connue de tous, bien qu'elle soit enveloppée d'une sorte de pudeur. Les textes, en effet, quoique dispersés dans l'espace et dans le temps, ne nous expliquent presque jamais ce qu'est une vi akanyā, se limitant à y faire allusion : signe que le public comprenait immédiatement de quoi il s'agissait.

3) Il ne s'agit pas d'une exclusivité du sexe féminin. Seule la višakanyā est créée artificiellement pour tuer, mais en Orient il existe aussi de nombreux cas d'êtres masculins analogues. Je ne citerai qu' un seul exemple : celui, extrêmement curieux, de Mahmud Shah, roi de Gujarat, vers le XVI ${ }^{\mathrm{e}}$ siècle, dont les voyages de Varthema et le livre de Duarte Barbosa nous ont laissé des témoignages. Ces deux textes ont été édités par Ramusio, qui a largement répandu cette légende en Europe. Voici par exemple ce que raconte l'Itinerario de Ludovico di Varthema (I470-1517), publié à Rome en I5IO et traduit dans presque toutes les langues d'Europe au cours des décennies suivantes :

El dicto Soldano ha li mostachi sotto el naso tanto longhi, che se li annoda sopra la testa come faria una donna le sue treze, et ha la barba biancha per fino alla centura; et ogni giorno mangia tossicho. Non crediate perbò che se ne empia el corpo, ma ne mangia una certa quantità; per modo che quando vuol far morire uno gran maestro, se lo fa uenire inanti spogiato et nudo [...] et appresso [...] buffa adosso a quella persona che nol far morire, per modo che in spatio di meza hora casca morta in terra. Questo Soldano tiene anchora tre o uero quatro milia donne, et ogni nocte che dorme con una, la matina se troua morta: et ogni uolta che ei se leua la camisa, mai più quella è tocchata da persona alchuna; et cossi li vestimenti noui. El mio compagno dimandò che cosa era, che questo soldano mangiaua cossi tosicho: resposero certi mercanti più uechi del soldano, ch'el patre lo hauea facto nutrire da piccolino de tosicho ${ }^{6}$.

Ce sultan a sous le nez de si longues moustaches qu'il les noue sur sa tête comme le ferait une femme avec ses tresses, et il a une barbe blanche qui descend jusqu’à sa ceinture; et chaque jour il mange un produit toxique. Mais n'allez pas croire qu'il en remplisse son corps; il en mange seulement une certaine quantité et quand il veut faire mourir un grand maitre, il le fait venir en sa présence dévêtu et nu $[\ldots]$ et ensuite $[\ldots]$ il souffle sur cette personne qu'il veut faire mourir, de sorte qu'en une demi-heure celle-ci tombe à terre, morte. Ce Sultan possède encore trois ou quatre mille femmes, et chaque nuit où il dort avec l'une d'elles,

6. Itinerario di Lodovico Varthema, nouvellement mis en lumière par A. Bacchi della Lega, Bologne, Romagnoli, I885, p. IOI et suiv. (nous traduisons). 
on la retrouve morte au matin : et chaque fois qu'il enlève sa chemise, celle-ci n'est plus jamais touchée par qui que ce soit; même chose pour ses vêtements. Mon compagnon demanda ce qu'était ce produit si toxique que mangeait ce Sultan : certains marchands plus vieux que le sultan répondirent que son père l'avait fait nourrir avec ce produit toxique quand il était tout petit.

Observons toutefois que l'image de l'homme venimeux existait aussi en Occident dans l'Antiquité; selon Antonino Liberale, Minos «éjaculait des serpents, des scorpions et des scolopendres, et toutes les femmes auxquelles il s'unissait, mouraient ${ }^{7}$ ", et il fut guéri par Procris avec une sorte de préservatif fabriqué à l'aide d'une vessie de chèvre. Puis, d'après ce que j'en sais, ce thème disparaît complètement : le seul exemple que je pourrais citer, à l'époque moderne, est peut-être le poème métaphorique et visionnaire de Baudelaire, "À celle qui est trop gaie», lequel, dans un paroxysme pervers, rêve de posséder une femme à travers «une blessure large et creuse» produite exprès dans son flanc pour lui infuser sa semence empoisonnée ${ }^{8}$.

4) Selon tous les témoignages, des plus anciens aux plus récents, il s'agit d'une coutume absolument réelle. Les chercheurs modernes ont souvent souligné le fait qu'il devait s'agir d'une pure fictio littéraire9, mais il en a été tout autrement jusqu'au seuil des temps modernes. Pour presque tous les auteurs qui ont écrit sur ce sujet et - nous pouvons le supposer - pour la plus grande partie de leur public, la puella venenata était une réalité et non une fantaisie. Il me semble même que certains textes orientaux - comme le traité sur les poisons de Sanâq, que j'ai consulté dans la vieille traduction de Müller montrent que l'on a plusieurs fois tenté de mettre en pratique cette légende, en soumettant de malheureuses enfants à un régime à base d'aconit, qui avait évidemment une issue fatale $\mathrm{e}^{\mathrm{IO}}$.

3. Ces textes indiens constituent le premier chapitre de l'histoire des femmes venimeuses. Le deuxième est entièrement l'œuvre des Arabes, lesquels, arrivant

7. A. Liberale, Metamorfosi, éd. G. Mordenti, Imola, La Mandragora, I998, chap. XLI, p. 94 (nous traduisons).

8. "Ainsi je voudrais, une nuit, / Quand l'heure des voluptés sonne, / Vers les trésors de ta personne, / Comme un lâche, ramper sans bruit, / Pour châtier ta chair joyeuse, / Pour meurtrir ton sein pardonné, / Et faire à ton flanc étonné / Une blessure large et creuse, // Et, vertigineuse douceur!/ À travers ces lèvres nouvelles, / Plus éclatantes et plus belles, / T'infuser mon venin, ma sœur!» (Ch. Baudelaire, "À celle qui est trop gaie», dans Les Fleurs du Mal [I86I], éd. Cl. Pichois, Paris, Gallimard, 1996, p. I85-186.

9. Par exemple Penzer, 1924, p. 313: "The poison-damsel herself has no existence in actual fact, but is merely the creation of the story [...]".

Io. Sanâq's Buch über die Gifte, dans A. Müller, "Arabische Quellen zur Geschichte der indische Medizin ", Zeitschrift der Deutsche Morgenländischen Gesellschaft, n 34, I880, p. 50I et suiv., p. 535 . 
en Inde à partir du viII ${ }^{\mathrm{e}}$ siècle, sont tellement frappés par ces étranges femmes venimeuses indiennes qu'ils ne se contentent pas, comme al-Qazwīnī, de les considérer comme une curiosité anthropologique digne d'attention, mais qu'ils les étudient dans une perspective typiquement scientifique. Grâce à eux, hors de l'Inde, l'histoire de la puella venenata fait son apparition dans deux domaines différents, mais interdépendants. D'un côté, nous la trouvons dans des ouvrages de toxicologie, à partir d'un traité du $\mathrm{IX}^{\mathrm{e}}$ siècle que nous connaissons dans la version pehlevi attribuée à Yahya ibn Khalid (mort en 806), vizir de Haroun el Rachid. De l'autre, elle affleure régulièrement dans les textes de médecine, à partir, d'après mes informations, de l'Al-hawi d'Abu Bekr ar-Razi (le Rhases des sources latines, mort en 932), un texte qui est traduit en latin en I279 et imprimé en 1506 sous le titre Liber [...] Helchauy, hoc est Continens artem Medicinae, dans lequel il est dit que "Quand les Éthiopiens veulent tuer des princes, ils nourrissent des jeunes filles avec du poison, de manière à ce que les plantes dont elles s'approchent sèchent et que leur crachat tue les poules et les autres bêtes, et les mouches qui volent près d'elles" ${ }^{\mathrm{II}}$. Cette information est reprise dans le Canon d'Avicenne ${ }^{\mathrm{I2}}$ (980-I037), qui l'attribue, sans fondement me semble-t-il, à Rufus d'Éphèse, un médecin grec de la seconde moitié du $\mathrm{I}^{\mathrm{er}}$ siècle après J.-C.; c'est vraisemblablement par ce biais que cette histoire arrive jusqu’à nous, quand Gherardo di Cremona (III4-II87) traduit ce qui restera, jusqu’au XVIII ${ }^{\mathrm{e}}$ siècle, le manuel de médecine de référence le plus répandu.

Sur la lancée du succès médiéval de la science arabe, l'histoire de la puella venenata se répand donc dans la culture européenne. Nous la retrouvons régulièrement, du XIII ${ }^{\mathrm{e}}$ siècle jusqu'au milieu du XVIII ${ }^{\mathrm{e}}$ siècle, dans une série disparate et très riche d'ouvrages scientifiques que l'on peut classer en plusieurs ensembles bien définis :

- Les ouvrages médicaux, qu'ils dépendent directement ou indirectement d'Avicenne (traductions, commentaires, etc.), ou qu'il s'agisse de traités généraux comme le Lilium medicinae de Bernard Gourdon ${ }^{13}$, les Disputaniones medicinae selectae de Matias Garcia ${ }^{14}$ ou l'Epistolarum medicinalium volumen tripartitum de Johann Lange von Lemberg ${ }^{15}$.

- Les traités de toxicologie, au moins à partir du célèbre Tractatus de venenis de Pietro d'Abano (I250-I316), plusieurs fois réimprimé jusqu'à la fin

II. Venetiis, I506, 1. XX, chap. 2, fo 4I3c (nous traduisons).

I2. Avicenne, Canon, translatus a magistro Gerardo Cremonensi, Venetiis, I490, liv. IV, fen. vi, Trac. I, chap. II, Sermo universalis de cautela a venenis, p. 691.

13. Bernardi Gordonii opus, Lilium medicinae inscriptum, Lugduni, apud Guilelmum Roillium, MDLIX, p. 53.

I4. Disputatio II, p. I33.

15. Liv. I, ep. I2, Francofurti, I589, 57 et suiv. 
du XVI ${ }^{\mathrm{e}}$ siècle, suivi par le De Venenis et antidotis d'Andrea Bacci (I520-I600), par le De Venenis et morbis venenosis de Girolamo Mercuriali ${ }^{16}$ ou par les Deux Livres des venins de Jacques Grévin (I539-1570). Remarquons que dans ce genre de littérature, l'histoire reste courante jusqu'au début du XVIII ${ }^{\mathrm{e}}$ siècle, comme le prouve le De Venenis in genere de Johann Linder, qui date de 1708.

- Les livres de botanique, comme le splendide Phytognomonica de Giovan Battista della Porta ${ }^{17}$ ou le Dryadum, Amadryadum Cloridisque Triumphus de Giorgio della Torre ${ }^{18}$, et des textes traitant différents sujets, comme la bizarre Sialologia historico-medica, hoc est Salivae humanae consideratio pshysico-medicoforensis de Martin Schurig' ${ }^{19}$, ou encore l'Opusculum de medicamentis bezoardicis de Johannis Juuenis ${ }^{20}$.

- Enfin, les recueils très populaires de curiosités et de merveilles, comme les Memorabilium centuriae d'Antoine Mizauld (mort en I578) 2I $^{21}$ les Lectionum antiquarum de Caelio Rodigino (I469-1525) ${ }^{22}$, les Neünhundert Gedächtnußwürdige Geheimniß und Wunderwerck de Georg Henisch (mort en 16I8) ${ }^{23}$ ou l'Artzney Kunt und Wunder Buch de Michael Bapst von Rochlitz (mort en $1603)^{24}$.

Cette constellation composite d'ouvrages scientifiques, qui assure sans solution de continuité la transmission de notre histoire du Moyen Âge jusqu'au seuil de l'époque moderne, considère toujours la puella venenata comme une réalité pure et indiscutable : si bien qu'en marge de cette histoire, on voit s'engager dans ces textes un débat enflammé sur la possibilité qu'un poison se transforme en nourriture. Très rares sont les auteurs qui s'écartent de la communis opinio et tiennent cette histoire pour fantaisiste ou impossible, comme Andrea Mattioli (1650-1577) dans ses magnifiques Commentarii in libros sex Pedacii Dioscoridis ${ }^{25}$ ou le grand Thomas Browne dans les Pseudodoxia epidemica ${ }^{26}$.

4. On est tellement persuadé qu'il existe une possibilité concrète de former une jeune fille venimeuse, qu'en élaborant l'histoire de la višakanyā, on forge

I6. Francofurti, apud heredes Andreae Wecheli, MDLXXXIIII, liv. I, chap. IX, An venena nutrire possint, p. 39.

17. Francofurti, I608.

I8. Patavii, 1685 .

19. Dresdae, Sumptibus haer. Miethii, I723, chap. v, De Salivae effectibus, \$8, p. 177 .

20. In Aegidius Everartus, De Herba Panacea, quam alii Tabacum vocant, Antverpiae, 1587 , p. 217.

2I. I, aphorismus 59, Lutetiae I566, bl. 9 b.

22. Libri xxx, Basileae, Froben, MDXLIII, L. XI, chap. XIII, p. 409.

23. Basel, 1575, p. 36.

24. Eißleben, I604.

25. Venetiis, In Officina Valgrisiana, MDLX, p. 716-717.

26. VII, chap. XVII.4 Of the woman fed with poyson that should have poysoned Alexander. 
des légendes pseudo-historiques pour expliquer la mort soudaine de souverains particulièrement portés sur les femmes. Ce fut le cas par exemple de Wenceslas II de Bohême (mort en I305), tué par voie vénérienne par sa mầtresse, selon Ottokar aus der Gaal27, et de Ladislas d'Anjou-Durazzo, dernier roi angevin de Naples, qui tenta vainement, avant César Borgia, de créer un état unitaire en Italie. Ladislas est à Pérouse et les Florentins craignent, non sans raison, qu' il veuille les attaquer.

[...] \& per quello havendo inteso che'l re stava innamorato della figlia d'un medico perugino, con la quale spesso si giaceva, è fama che hauessero con gran somma de danari subornato il medico che per mezzo della figlia l'avesse avelenato, \& che'l medico indutto dall'avaritia, anteponendo il guadagno alla vita della figlia, l'avesse persuasa ad ungersi le parti genitali d'una untione pestifera quando andava a star co'l re, perch'era compositione atta a dare tal diletto al re nel coito, che non havrebbe potuto mai mancare dal amor suo, et che per questo il re se infermò d'un male al principio lento, \& incognito. [...] Aggravatosi il male, \& partendosi il re di Perugia, per venirsi ad imbarcare sulle Galee ad Ostia [...], se imbarcò assai grave del male, [...] et gionto a Napoli a due di agosto dell'anno MCCCCXIII, [...] il sesto di di Agosto usci di vita con fama di mal cristiano $[\ldots]^{28}$

[...] \& pour cela, ayant su que le roi était amoureux de la fille d'un médecin de Pérouse, avec laquelle il couchait souvent, on dit qu'ils ont suborné avec une grande somme d'argent ce médecin, lequel l'a empoisonné par l'intermédiaire de sa fille, \& que ce médecin, poussé par la cupidité et faisant passer le gain avant la vie de sa fille, avait persuadé celle-ci d'oindre ses parties génitales avec un onguent pesteux quand elle couchait avec le roi, car c'était une composition qui donnait un tel plaisir au roi dans le coït qu'il ne pourrait jamais se passer de son amour; pour cette raison, le roi tomba malade d'un mal lent au début, \& inconnu. [...] Le mal s'étant aggravé, \& et le roi ayant quitté Pérouse pour venir s'embarquer sur les Galères à Ostie [...], il s'embarqua fort atteint par ce mal, [...] et arrivé à Naples le deux août de l'an MCCCCXIII, [...] le sixième jour d'Août il quitta la vie avec la réputation d'un mauvais chrétien [...]

Bien entendu, cet onguent est «sugo di Napello, prestantissimo veleno» («le suc de l'aconit, poison exceptionnel») (Pandolfo Collennuccio); mais pour d'autres (selon le témoignage de Muratori), il s'agissait simplement d'une grave infection vénérienne "que lui avait passée, à en croire la rumeur, une putain...» (Annali d'Italia ad an. I4I4). Il est significatif que l'historiographie orientale connaisse elle aussi des histoires de ce genre.

27. Voir Ottokar aus der Gaal, Steirische Reimchronik, dans MGH, Deutsche Chroniken, vol. 5, t. II, p. II27 et suiv. Fait intéressant, Ottokar assimile l'assassinat de Wenceslas à la coutume en usage chez les Romains, lesquels, dit-il, élevaient une petite fille «de sorte que dans sa bouche il y ait du poison".

28. A. di Costanzo, Historia del regno di Napoli, Nell'Aquila appresso Gioseppe Cacchio, I58I, livre XII, p. 279-280 (nous traduisons). 
5. La tradition culturelle arabe introduit cette histoire en Europe également à travers une variante au ton plus littéraire, répandue à travers un ouvrage qui connaît un immense succès pendant un demi-millénaire : le Kitâb sirr al-asrâr ( Livre de la science de gouvernement»), œuvre d'un auteur arabe inconnu (qui affirme d'ailleurs élaborer une source grecque), datant vraisemblablement du $\mathrm{x}^{\mathrm{e}}$ siècle. Rapidement traduit dans une myriade de langues - et en particulier en latin, sous le titre, des plus séduisants, de Secretum secretorum qui reprend néanmoins littéralement le titre arabe cité plus haut et littéralement traduit -, ce traité, qui se présente sous la forme d'une longue lettre d'Aristote à Alexandre, est aujourd'hui totalement inconnu hors du cercle des spécialistes du Moyen Âge, mais il a sans doute été - pour reprendre les termes de Lynn - "the most popular book in the Middle Ages ${ }^{29} »$. Objet d'une véritable vénération et attribué à Aristote, le Secretum secretorum a été inlassablement copié pendant des siècles (il existe plus de 600 manuscrits de la version latine...), il a été étudié, élaboré, traduit et commenté. Bien entendu, le prestige extraordinaire de ce texte et sa fortune persistante ne pouvaient que se refléter sur les récits qu'il contenait : et en particulier, en ce qui nous concerne, sur notre histoire, rapportée au chapitre xxI de l'édition Steele ${ }^{30}$ et significativement intitulée De cavendis mulieribus et venenis et ab omni immunidicia. Je la cite dans la version italienne du Reggimento dei Signori, la plus ancienne version du Secretum secretorum :

Alexandro, ricorditi de' facti del regno d'India, come a te molti doni e molti presenti facti furo, per cagione di buona amistade, e altri molti nuovi doni, intra i quali mandata fu a te quella istraina e nuovissima pulcella, la quale ripiena e nodrita fu da la sua infantia di veneno di serpente. Et se non fusse che io in quell'ora sagacemente guardai in lei, e per arte di sopra io connovi et vidi et judicai ciò ch'ella cusi audacemente, sensa lo suo volto cessare o ver mutare, di nulla vergogna mostrando, lo volto suo in delle facce alli nomini ficcava, dèi pensare in del tuo animo veramente, ch'ella avrebbe morti li huomini solo col suo mirare u del vero col mordimento; la qual cosa puoi tu, per diricto experimento provasti et trovasti, et se questo certissimamente io mostrato non t'avesse, morto t'arebbe in de l'ardore del carnale dilecto seguire ${ }^{31}$.

Alexandre, souviens-toi des faits du royaume de l'Inde, que l'on te fit beaucoup de dons et de cadeaux, par raison de bonne amitié, et bien d'autres étonnants cadeaux, parmi lesquels on t'envoya cette étrange et prodigieuse jeune fille, laquelle fut

29. L. Thorndike, A History of Magical and Experimental Science, New York, I923, vol. II, p. 267.

30. Secretum secretorum cum glossis et nutulis, nunc primum edidit Robert Steele, accedunt versio anglicana ex arabico edita per A. S. Fulton, version vetusta Anglo-normanica, nunc primum edita, Oxonii, e Typrograpeo Clarendoniano (Oxford University Press), 1920, p. 60.

3I. Je cite ce passage d'après A. d'Ancona, Il tesoro di Brunetto Latini versificato, dans Atti della R. Accademia dei Lincei, Serie Quarta, Classe di Scienze morali, Storiche e Filologiche, vol. IV, par. I, Memoria, p. III-274, Rome, I888, p. I4I, n. 4. 
remplie et nourrie depuis son enfance de venin de serpent. Et si, à ce moment-là et je ne l'avais moi-même examinée avec sagacité, et si par un art supérieur je n'avais connu et vu et jugé qu'elle fixait son regard sur le visage des hommes [...] sans cesse, sans bouger et sans montrer nulle honte [...], tu dois vraiment penser en ton esprit qu'elle aurait tué les hommes rien qu'en les regardant ou en les mordant; laquelle chose tu as constatée et vue par expérience directe, et si je ne te l'avais pas montré, il est certain qu'elle t'aurait tué dans l'ardeur du plaisir charnel.

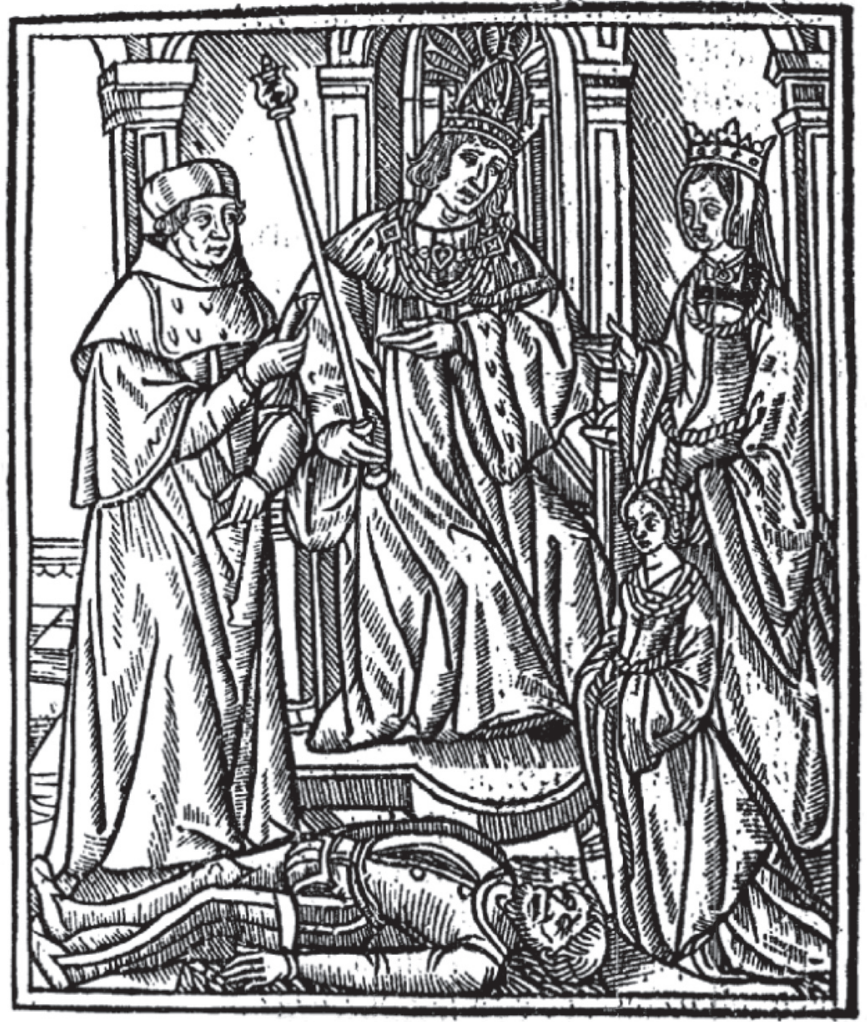

Fig. 1 - Pierre Gringore, Les Fantasies de Mère Sote, imprimées à Paris par Jehan Petit, 1516 - Alixanre, Aristote et la pucelle venimeuse, fo $8 \mathrm{r}^{\mathrm{O}}$.

On pourrait suivre longtemps la fortune de cette petite histoire dans la littérature européenne : depuis les nombreuses versions vulgaires du Secretum - en castillan, catalan, hébreu, russe ancien, ancien français et ainsi de suite -, jusqu'aux innombrables élaborations du récit qui se trouvent dans des ouvrages appartenant à tous les genres : je cite, presque au hasard, les Proverbes de Guilhem de Cervera (XıII ${ }^{\mathrm{e}}$ siècle), le Frauenlob de Heinrich von Meissen (vers I250-I3I8), l'Alexander Geesten de Jacob van Maerlant (vers I257), le Compendiloquim et le Communiloquium de Johannes Gallensis (milieu du 
XIV $^{\mathrm{e}}$ siècle), Der Renner de Hugo von Trimberg, Les Fantasies de Mère Sote de Pierre Gingore (I516, fig. I) - jusqu'au De Animalibus d'Albert le Grand, au Liber de introductione loquendi de Filippo da Ferrara ou à la Sylva sylvarum de Francis Bacon (Centuria V, $\$ 499$, p. 4I7). Comme il n’est pas possible d'examiner toute cette production, même dans ses grandes lignes, je me contenterai d'ajouter deux observations rapides en marge de cette liste.

- En premier lieu, tout en conservant le ton «historique» de la narration (c'est-à-dire en présupposant la réalité de l'histoire qu'ils racontent), ces textes introduisent des expansions narratives, parfois assez libres et non dépourvues d'un certain intérêt. Ainsi, dans la version du Placides et Timéo, qui date du $\mathrm{XIV}^{\mathrm{e}}$ siècle, Alexandre est accompagné d'Aristote et de Socrate. Le roi qui a créé la jeune fille venimeuse l'expérimente prudemment sur un ennemi, lequel est tué quand il tente de l'embrasser, et les deux philosophes, pour convaincre Alexandre du danger que représente la jeune fille, ordonnent à un serviteur de l'embrasser, ce qui provoque évidemment la mort immédiate du malheureux.

- Ensuite, et surtout, toute cette production remplace régulièrement le thème végétal par le thème serpentin : la poison maiden devient telle non pas grâce à l'aconit mais parce que "ab infancia imbuta et nutrita fuit veneno serpentum, ita quod sua natura conversa fuit in naturam serpentum» ("elle a été imbibée et nourrie depuis son enfance de venin de serpent, de sorte que sa nature en a été transformée en nature serpentine»). Cette substitution extrêmement significative devient même paroxystique dans certains textes. Je me réfère en particulier à une traduction versifiée en langue vulgaire du Trésor de Brunetto Latini, remontant au XIV ${ }^{\mathrm{e}}$ siècle, où notre puella est déposée aussitôt après sa naissance dans un énorme œuf de serpent et élevée par le reptile qui la nourrit "di quelli elementi / che nutricò li altri serpenti» ("de ces aliments / avec lesquels il nourrit les autres serpents»); non seulement elle devient horriblement venimeuse, mais elle perd tout vestige d'humanité, de sorte que "non parlava / ma come uno serpente sifolava» ("elle ne parlait pas / mais sifflait comme un serpent»), et ce n'est qu'au terme d'une longue domestication qu'elle acquiert la parole et des habitudes humaines ${ }^{32}$. Il vaut la peine d'observer que ce que j'ai appelé le thème végétal est préférable de tous les points de vue au thème ophidien : d'abord, parce que c'est le thème "authentique», attesté par toute la tradition orientale, et ensuite parce que, d'une part, l'Aconitum napellus apparaît plus logique, si l'on peut dire, d'un point de vue toxicologique, car il empoisonne aussi par contact, comme notre puella, contrairement au serpent, qui injecte son venin; et, d'autre part, parce qu'il est plus

32. D’Ancona, I888, p. 137 et 139. 
motivé d'un point de vue esthétique, la belle plante mortelle correspondant parfaitement à la belle jeune fille mortifere. Pourtant, la protagoniste de nos récits, qui est à l'origine une splendide fleur toxique, devient un reptile - «de natura de las bivoras» (" de la nature des vipères»), selon la Poridad de las poridades, version médiévale espagnole du Secretum ${ }^{33}$ - et elle le devient au point qu'elle transmet son venin "solo morsu vel visu» (Steele, p. 60), "seulement par la morsure ou le regard» ou, pour citer les termes de Grégoire le Grand, «et cujus morsu moriebantur homines sicut ex morsu serpentium : et humor salivalis in ipsa fuit venenum ${ }^{34}$ " ("et par sa morsure les hommes mouraient comme de la morsure des serpents; et même la salive en elle était un poison»).

6. À ce stade, le philologue a accompli sa tâche : Wilhelm Hertz, suivi par tous ceux qui, comme moi, ont pillé ses très doctes pages, se limite à présenter une honnête vue d'ensemble de cette production littéraire impressionnante, sans rien ajouter d'autre. Mais il me semble que c'est précisément de là que nous devrions partir, en nous posant un certain nombre de questions. Par exemple, pourquoi l'histoire de la puella venenata a-t-elle eu en Occident un succès si durable et si immensément vaste? Pourquoi, malgré ce succès, le thème masculin correspondant, qui existait pourtant pendant l'Antiquité et qui est souvent présent en Orient, disparaît-il complètement? Pourquoi, surtout, le thème serpentin s'affirme-t-il résolument à côté du motif végétal de l'aconit? Et en dernier lieu, que signifient et qu'enseignent ces récits, pris dans leur ensemble?

Il me semble que la philologie ne peut pas répondre à ces questions : alors que l'anthropologie pourrait le faire, ou mieux encore, peut-être, une discipline qui parvienne à réunir les deux perspectives dans une vaste synthèse : la mémétique, peut-être, si nous arrivons à la développer correctement.

Le problème est, me semble-t-il, que l'histoire de la puella venenata n'est pas particulièrement remarquable en soi, même dans ses versions les plus ouvertement narratives. Elle le deviendra seulement dans certaines élaborations artistiques : par exemple, dans le Mandragore de Machiavel ou dans ce cauchemar gothico-lesbien qu'est Christabel de Coleridge. Cette histoire connaît pourtant un succès extraordinaire pendant des siècles parce qu'elle exprime certaines des peurs masculines les plus profondes. Nous ne devons pas oublier, en effet, que pendant toute la période qui nous intéresse, les femmes

33. L. A. Kasten (dir.) Poridad de las poridades, Madrid, Seminario de estudios medievales españoles de la universidad de Wisconsin, I957, p. 4I.

34. B. Alberti Magni, Operum, t. VI, Lugduni, I65I, p. 236, De Animalibus, liv. VII, tractatus ii, chap. v, p. 405 b. 
sont des bêtes venimeuses : des êtres qui distillent chaque mois, comme les serpents, avec leurs menstruations, l'un des venins les plus horribles que le monde connaisse et qui se purifient et se renouvellent chaque mois à travers cette alchimie, comme les serpents. Pour s'en convaincre, il suffit de lire Pline, Isidore, le De secretis mulierum d'Albert le Grand, et beaucoup d'autres ouvrages de même teneur, jusqu'à un petit traité du premier quart $\mathrm{du} \mathrm{XVIII}^{\mathrm{e}}$ siècle, la Parthelonogia historico-medica, hoc est Virginitatis consideration de Martin Schurig ${ }^{35}$, qui consacre un chapitre entier au sang menstruel, "pessimus et venenatus» (" très nuisible et empoisonné»). Schurig rappelle, avec Van Helmont, que celui-ci "per gradus ad liquaminis cadaverosi malignitatem ascendens assumit veneni indolem» (p. 239) ("en s'approchant graduellement de la pernicieuse sanie des cadavres, parvient à la nature du poison ") et démontre par de nombreux exemples sa terrible dangerosité. Quant au dominicain Felix Fabri (I438 ou I439-I5O2), auteur de l'Evagatorium dans lequel il décrit la Crète en affirmant que cette île est dépourvue d'animaux venimeux, il observe :

Verum unum genus animalis habet haec regio commune cum aliis regionibus, cujus morsus est venenatus et mortiferus. Dicunt enim, quod si mulier irata virum dentibus mordet aut unguibus lacerat, statim veneno infectus moritur, ac si morsus pessimae bestiae fuisset laesus ${ }^{36}$.

En vérité, cette région a en commun avec les autres régions un type d'animal dont la morsure est empoisonnée et mortelle. Ils disent en effet que si une femme en colère mord avec les dents ou lacère un homme de ses ongles, celui-ci meurt, infecté par le venin, comme s'il avait été blessé par la morsure de la plus terrible des bêtes.

À partir de ces idées, extrêmement anciennes, inextirpables et incroyablement répandues, nous pouvons peut-être comprendre pourquoi une analogie fondamentale et une étroite affinité sont apparues entre la femme et le serpent en des temps très reculés; une analogie et une affinité que la tradition juive trouve dans le mythe de la chute, qui se manifeste à l'époque classique à travers des déesses serpentiformes ou dotées de traits ophidiens - comme Méduse, pour ne citer qu'un seul exemple connu de tous - et qui alimente à partir du Moyen Âge un nombre vraiment impressionnant de thèmes mythiques, folkloriques, narratifs et figuratifs : songeons, pour ne citer qu'un seul exemple, à la semi-ophidienne Mélusine ${ }^{37}$.

35. Dresdae et Lipsiae, apud Christophori Hekelii, I729.

36. Frater Felic quis Fabri Evagatorium, éd. Hassler, Stuttgartiae, I849, II, p. 280-28I.

37. J'ai examiné ce problème dans un article qui peut être considéré de plusieurs points de vue comme propédeutique à celui-ci : C. Donà, "La metamorfosi segreta : dalla donna serpente alla puella venenata», dans Fr. Zambon (dir.), "Viridarium» : La metamorfosi, n ${ }^{\circ} 6$, Milan, Medusa - Venise, Fondazione Giorgio Cini, 2009, p. 47-8o. 
Le charme dangereux et fatal de la femme serpent a toujours été perçu et célébré par l'art occidental; et il a été assidûment cultivé, ajouterais-je, étant donné que la plupart des bijoux féminins antiques qui nous sont parvenus ont une forme serpentine et qu'aujourd'hui encore, une certaine féminité agressive et "pythonienne» a de nombreux amateurs. Mais ce charme est perçu comme quelque chose d'extrêmement dangereux qui mêle de façon troublante et inquiétante la séduction et la mort. Hans Baldung Grien l'a exprimé de la manière la plus claire qui soit en I 515 dans un tableau montrant une Ève nue et provocante qui noue un pacte funeste avec une Mort diabolique et corrompue et avec un serpent à l'air fourbe et satisfait; en souriant sournoisement, Ėve cache, derrière son dos, la pomme fatale...

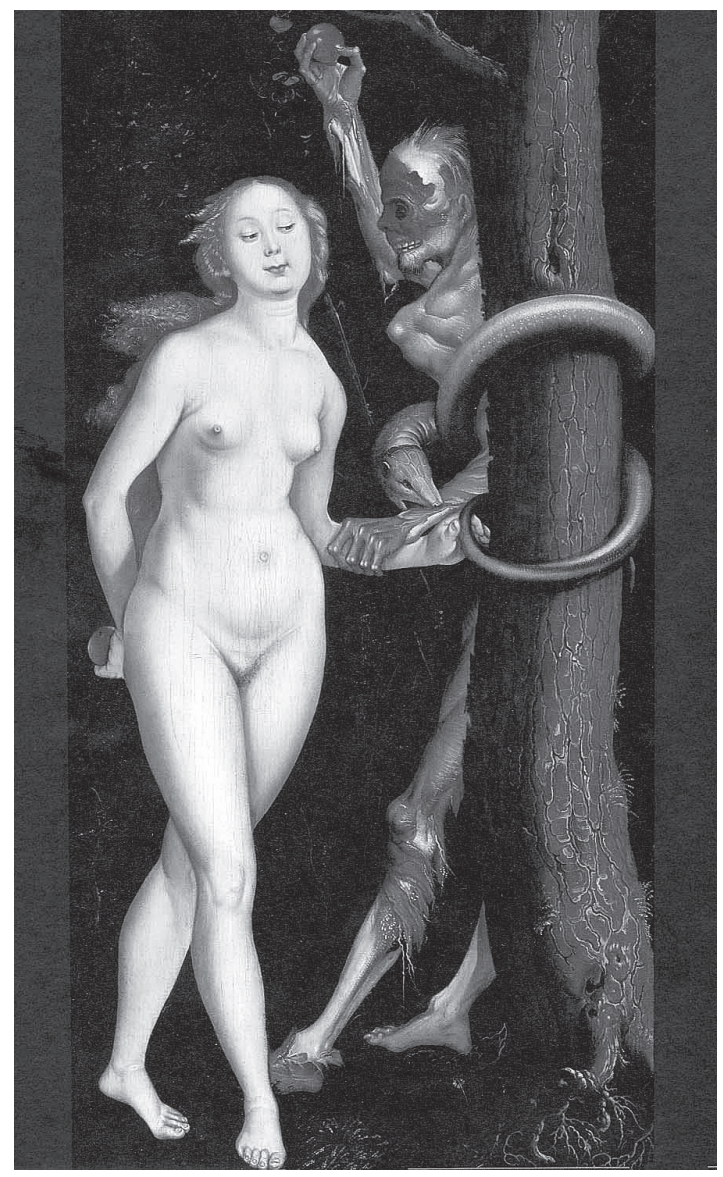

Fig. 2 - Hans Baldung, Ève, la mort et le serpent, huile, Ottawa, National Gallery of Canada. 
Pour de nombreuses raisons, cette affinité entre les femmes et le serpent s'exprime essentiellement à travers la sexualité, et même, plus explicitement, dirais-je, par la voie vaginale : on peut sans doute comprendre ce fait, à travers l'équivalence venin = sang menstruel, mais aussi en raison d'une certaine ressemblance entre l'organe féminin et la bouche grande ouverte du serpent prêt à mordre.

Dans l'imaginaire masculin, du reste, le vagin est souvent perçu comme un lieu dangereux : par exemple, dans le thème du vagin denté, extrêmement répandu dans le mythe, mais aussi dans le folklore moderne ${ }^{38}$. Dans les versions rationalisées, le danger est causé par les femmes. Ainsi, Albert le Grand met en garde contre les mulieres qui infligent aux hommes des blessures inguérissables en introduisant dans leurs propres corps des morceaux de fer :

Nota quod quadam mulieres sunt ita cauta et astuta, et accipiunt ferrum et apponunt vulua, et illud ferrum ledit virgam sed vir non percipit propter nimiam delectationem et dulcedinem vulva, postea tamen sentis : ideo maxime cauendum est ne fiat coitus cum mentruosis mulieribus, quia exinde homo posset incidere in lepra, et quandoque in magnam infirmitatem ${ }^{39}$.

Remarque que certaines femmes sont si rusées et si astucieuses qu'elles prennent un morceau de fer et le placent dans leur vagin et ce fer blesse la verge de l'homme sans qu'il s'en aperçoive, pour le grand plaisir et la douceur de la vulve. Mais après, il le sent. À ce propos, il faut surtout se garder d'avoir un coït avec des femmes réglées car l'homme peut en contracter la lèpre et parfois une grave infirmité.

De manière analogue, on racontait parmi les soldats américains l'histoire de prostituées liées aux Viêt-congs qui introduisaient dans leur vagin des lames de rasoir et des éclats de verre pour blesser leurs clients américains ${ }^{40}$. Certains lecteurs se souviendront peut-être en parallèle d'une scène mémorable de Cris et chuchotements d'Ingmar Bergman.

Quand la narration se déplace au niveau du mythe, la dangerosité mortifere de la femme peut être expliquée de différentes manières : par exemple, elle peut être protégée par un amant surnaturel qui tue ceux qui essayent d'avoir un commerce avec elle, comme l'Asmodée du Livre de Tobie, qui est d'ailleurs le «fils du dragon»; ou alors, plus simplement, le vagin devient la tanière d'un serpent que la femme porte en elle. Il s'agit du motif F 582 de l'index Thompson, Serpent Damsel - Woman has serpent inside which come

38. Voir par exemple V. Elwin, «The Vagina Dentata Legend», British Journal of Medical Pscychology, $\mathrm{n}^{\circ}$ 19, 1943, p. 439-453; R. Lionetti, L. Iovine, 32 simboli. Denti, morsi e vagine dentate, Trieste, Edizioni della Libreria Einaudi, 2004.

39. Albert le Grand, De Secretis mulierum [1580], p. 55.

40. M. Gulzow, C. Mitchell, "Vagina dentata" and "Incurable Venereal Disesase” : Legends of the Viet Nam War", Western Folklore, n 39, 1980, p. 306-316. 
out and kills her bridegrooms, qui caractérise surtout les fables du type AT 507, The Serpent Maiden. C'est dans ces fables, étudiées par Gordon Hall Gerould, Sven Liljeblad, Elwin et d'autres chercheurs encore, que le thème de la puella venenata trouve sa réalisation la plus spectaculaire et expressive : chaque nuit, un serpent venimeux et famélique sort des héroïnes de ces histoires et se jette sur leurs malheureux amants, qu'il tue sans merci. Seul peut survivre à cette épreuve un homme particulièrement sagace qui ne s'abandonne pas à l'amour, mais attend patiemment la sortie du reptile pour le tuer et pour purifier la femme (des autres serpents qu'elle porte souvent en elle, radicalement, en la coupant en deux). J'en cite un exemple ethnique, une histoire des Shuswap relatée dans le beau livre de Roberto Lionetti et Luisa Iovine sur le vagin denté :

L'[eroe] vide una donna che gridava e si lamentava: "Chi vuole dormire con me?" Dopo essersi messo in bocca una foglia che masticava, [egli] andò a dormire con lei. Vide sparse attorno molte ossa umane. Tutti gli uomini che avevano dormito con lei erano morti, poiché gli organi intimi della donna non erano altro che un serpente... Egli sputò la foglia che aveva masticato sui suoi organi e li trasformò, dicendo: "D'ora in poi le donne non uccideranno più gli uomini con cui hanno rapporti sessualit".

Le [héros] vit une femme qui criait et se plaignait : "Qui veut dormir avec moi? ». Après avoir mis dans sa bouche une feuille qu'il mastiqua, [il] alla dormir avec elle. Il vit beaucoup d'os humains éparpillés alentour. Tous les hommes qui avaient dormi avec elle étaient morts, car les organes intimes de la femme n'étaient autres qu'un serpent... Il cracha la feuille qu'il avait mastiquée sur ses organes et il les transforma, en disant : «Dorénavant, les femmes ne tueront plus les hommes avec lesquels elles ont des rapports sexuels.»

Certaines images, provenant de cultures très différentes, montrent l'ancienneté et la grande diffusion de ces idées, mais aussi la ténacité avec laquelle elles parviennent à survivre dans l'imaginaire collectif. Voici, respectivement, un pendentif en argent provenant de Ras Shamra (XIII ${ }^{\mathrm{e}}$ siècle avant J.-C., fig. 3), une sculpture romane provençale du XII ${ }^{\mathrm{e}}$ siècle (fig. 4), et le dessin d'un psychotique contemporain (fig. 5).

4I. R. Lionetti, L. Iovine, 32 simboli. Denti, morsi e vagine dentate, ouvr. cité, p. II. 


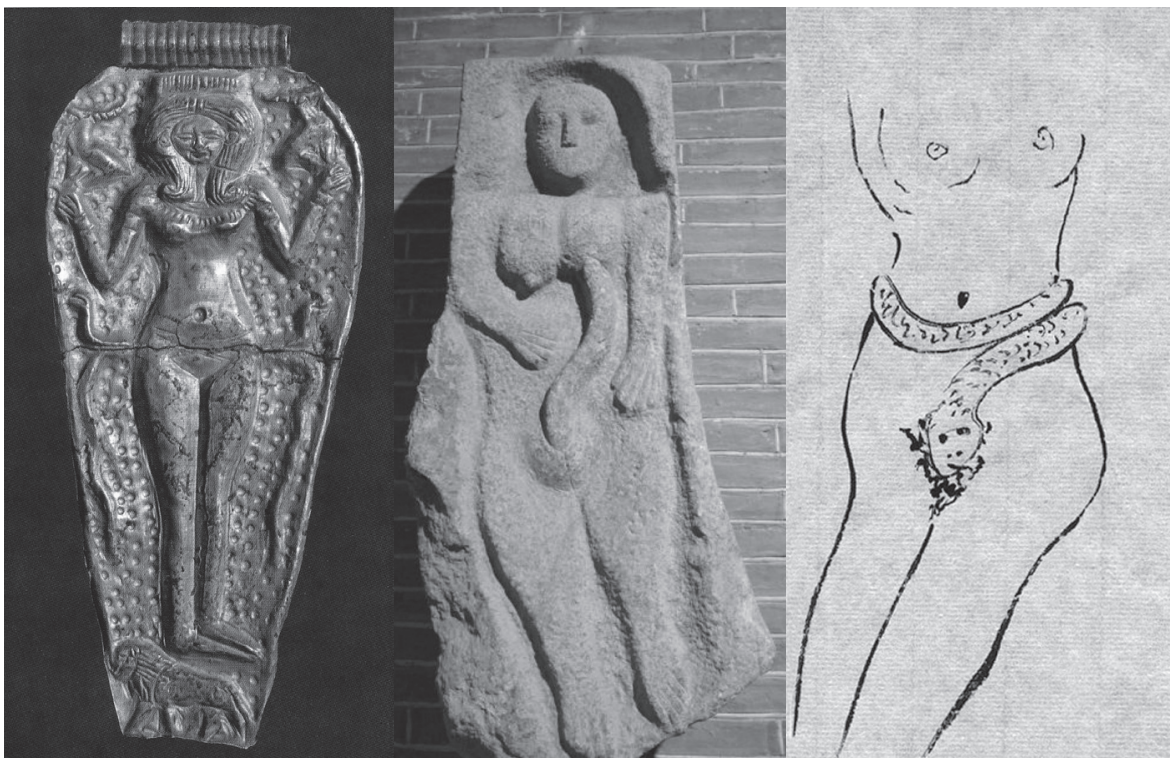

Fig. 3 - Déesse aux serpents, pendentif en or, Rās Šamra, $\mathrm{XV}^{\mathrm{e}}$ siècle avant J.-C., musée du Louvre.

Fig. 4 - Vallée d'Oô, stèle du haut Moyen Âge avec femme et serpent, Toulouse, musée des Augustins.

Fig. 5 - Dessin de psychotique.

Carolee Schneemann, dans sa performance Interior Scroll, réalisée en 1975, extrayait un serpent de son sexe, et décrivait son point de vue en ces termes :

I saw the vagina as a translucent chamber of which the serpent was an outward model: enlivened by it's passage from the visible to the invisible, a spiraled coil ringed with the shape of desire and generative mysteries, attributes of both female and male sexual power. This source of interior knowledge would be symbolized as the primary index unifying spirit and flesh in Goddess worship $p^{42}$.

J'ai considéré le vagin comme une chambre translucide dont le serpent était un modèle extérieur : animé par son passage du visible à l'invisible, un anneau en spirale entouré par la forme du désir et des mystères génératifs, les attributs de la puissance sexuelle féminine et masculine. Cette source de connaissance intérieure serait symbolisée par le principal index unifiant l'esprit et la chair dans le culte de la déesse.

La diffusion universelle de croyances de cette teneur peut être démontrée par une belle page de ce best-seller européen que fut le livre de Mandeville,

42. C. Schneemann, "The Obscene Body/Politic», Art Journal, n 50, 4, Censorship II (Winter, I99I), p. 28-35. 
selon lequel, dans une île d'Extrême-Orient, on a coutume de louer les services d'un tiers pour déflorer les femmes la première nuit de noces.

[...] quar cils du pays tiegnent a si grande chose et a si perillouse a despuceller une femme qe lour semble qe cils qe les despucelle se met en aventure de morir. Et si ly maritz troeve sa femme pucelle l'autre nuyt après qe ly autre ne l'eust despucellé pur yveroigne ou pur autre cause, il soy pleindroit du vallet qe n'averoit mie fait soun dever, aussy bien come si ly vallet ly vousist tuer. [...] Et jeo fiz demander la cause pur quoy homme tenoit celle custume, et homme me dit qe [fo $\left.96 \mathrm{v}^{\circ}\right]$ aunciennement ascuns avoient esté mortz pur femmes despuceller qe avoient serpentz el corps, et pur ceo tiegnent ils celle custume et font toutdis assaier le passage a un autre avant q'ils se mettent en aventure $[\ldots]^{43}$

[...] mais les gens de ce pays considèrent qu'il s'agit d'une chose si grave et si dangereuse, c'est-à-dire déflorer une femme, parce qu'ils croient que ceux qui la déflorent se mettent en danger de mourir; et si la deuxième nuit les maris ne trouvent pas leurs femmes déflorées, ils se plaignent de leur valet, lequel n'a pas fait son devoir, comme si ce serviteur avait voulu les tuer [...]. Nous leur demandâmes pour quelle raison ils conservaient une telle coutume; et ils répondirent qu'en déflorant des femmes, autrefois plusieurs hommes étaient morts; elles avaient semble-t-il des serpents dans leur ventre. C'est pour cette raison qu'ils conservent encore cette coutume et font toujours essayer le passage à quelqu'un avant de tenter l'aventure $[\ldots]$

C'est sûrement sur le fond de ces obsessions - nous pourrions les appeler "complexe de la mante religieuse» - que nous pouvons comprendre, et dans une certaine mesure expliquer, le succès durable de l'histoire de la puella venenata : celle-ci traverse certaines des zones les plus obscures et douloureuses du psychisme et elle exprime de la manière la plus inquiétante et la plus profonde certaines obsessions masculines persistantes sur le sexe et la mort, c'est-à-dire sur deux faisceaux conceptuels d'une importance extraordinaire.

43. J. de Mandeville, Le Livre des merveilles du monde, éd. Ch. Deluz, Paris, CNRS, 20oo, p. 449-450. 